\title{
Samples of the Collection of Old Medicine Raw Materials of the Department of Pharmacognosy of St. Petersburg Chemical Pharmaceutical University, Which Can Be Used in Food
}

\author{
A. V. Klemper \\ Pharmacognosy Department of St. Petersburg Chemical-Pharmaceutical University, Professor Popov Street, 4/6, St. Petersburg \\ 197277, Russia
}

\begin{abstract}
In the world there are many collections of the pharmaceutical trend. In America, the exhibits of this profile are in the Smithsonian Museum of Natural History in Washington, in Europe these are collections of pharmacy museums in Heidelberg (Germany), Krakow and Warsaw (Poland), Basel (Switzerland), Riga (Latvia), and others. Some of them have many thousands of exhibits. There are also separate collections of pharmaceutical items for educational institutions, for example, a collection of old medicinal raw materials in Vigan's Cabinet, Queen College, Cambridge (Great Britain). All these meetings, however, have long been known and described in detail. Most of them do not have a strict orientation and are presented, along with old medicinal raw materials, as well as tools and appliances, dishes, books, herbaria and various auxiliary items. All the more interesting is the collection indicated in the title of the article, which arose literally from non-existence. She was transferred to the educational institution at its creation, and this year she, like him, turns a hundred years old. It has a strict focus and is represented almost exclusively by medicinal raw materials. Studying the samples of this old collection, as a whole of medical profile, makes it possible to understand that many plants, which initially had only food use, gradually became pharmaceutical objects. Probably, it was the long practice of food use, with the fixing of associated pharmacological effects in the memory, that became the reason for choosing certain types of raw materials as medicinal. Ordinary foods, protein and starchy, began to be perceived as strengthening, mucous-as enveloping and anti-ulcer, some fruits and herbs—as antiscorbutic, sharp—as appetizing and improving the work of the stomach, and so on.
\end{abstract}

Key words: Food, medicinal raw materials, collection, old samples.

The pharmacognosy department of the St. Petersburg Chemical-Pharmaceutical University (SPCPU) holds a collection of medicinal raw materials, most of which are dated to the second half of the 19th/early 20th centuries. The earliest specimens are dated 1829. Despite the representative size, more than three thousand units, it is unknown neither in Russia nor abroad. No mention was made of it in the literature. The first article, in connection with

Corresponding author: Aleksey Klemper, candidate of pharmaceutical sciences, associate professor, research fields: medicinal herbal raw materials and related environmental aspects. the beginning of work with the collection, was published three years ago [4].

The origin of the samples is diverse-Europe, Caucasus, Central Asia, Siberia, Far East, Southeast Asia and New Guinea, India, Ceylon, North, North, Central and South America, Africa, Madagascar, Australia and the islands near them. Vegetable raw materials are grouped according to morphological groups: underground organs (roots, root crops, rhizomes, tubers), bark, grass, leaves, flowers, fruits, seeds. Species (or other groups, if the exact definition is impossible) of which the samples are derived, are more than 1,200. The most popular are chin bark, 
coffee seeds, senna leaves, rhubarb, extracts of catechu and aloe, galls, gum-resin, essential oils, tea production samples. Not all samples are medicinal raw materials. Some were collected for scientific or purely collectible purposes. This article discusses samples that can be used in cooking directly or as additives.

The authentic name of the sample, by label or enclosed piece of paper, is given in quotes. A horizontal line (|) separates the words separated in the label by meaning and in different lines from each other. The text without quotes refers to a sample without inscriptions, defined by external signs. In case of impossibility of the exact species, clan, etc., the assignment is given the name of the smallest systematic group into which the species belongs. Modern Latin names for plants and mushrooms are verified by Plant List and Mycobank.

Conventionally, the samples are divided into commonly used raw materials (Table 1) and non-commonly used raw materials (Table 2). For common raw materials, references to the source of information are not given. In the presence of a number of similar samples, the name of one of them is selectively given. Words that do not have a translation are given by transliteration, in square brackets, for example, “Кумиэн”__[Cumien]”.

Table 1 Commonly used raw materials.

\begin{tabular}{|c|c|c|}
\hline № & Sample name & $\begin{array}{l}\text { Names of species and family } \\
\text { (part or product is indicated in brackets) }\end{array}$ \\
\hline \multicolumn{3}{|c|}{ Custom raw materials } \\
\hline 1 & “Anacardium occidentale Herm. | Feuerwerks-nü $\int$ se” & Anacardium occidentale L., Anacardiaceae (fruits, seeds) \\
\hline 2 & Peanut beans & Arachis hypogaea L., Fabaceae (fruits) \\
\hline 3 & “Avena sativa Linn. (Oats)” & Avena sativa L., Poaceae (fruits) \\
\hline 4 & “Crab | [Ditz Srin]” & Brachyura (claws) \\
\hline 5 & Shrimps & Caridea (whole) \\
\hline 6 & "Fructus Fragariae" & Fragaria vesca L., Rosaceae (fruits) \\
\hline 7 & "Soy seeds yellow" & Glycine max (L.) Merr., Fabaceae (seeds) \\
\hline 8 & "Ervum Lens Linn. (Seeds, Split,Variety)” & Lens culinaris Medik., Fabaceae (seeds) \\
\hline 9 & “Fructus Oleae Europeae” & Olea europaea L.,Oleaceae (fruits) \\
\hline 10 & “Oryza sativa Linn. | Dudhkalam (Husked)” & Oryza sativa L., Poaceae (fruits) \\
\hline 11 & "Panicum miliaceum Linn. (Seeds)" & Panicum miliaceum L., Poaceae (fruits) \\
\hline 12 & “Semen Phaseoli” & Phaseolus sp., Fabaceae (seeds) \\
\hline 13 & "Phoenix dactilifera” & Phoenix dactylifera L., Arecaceae (fruits) \\
\hline 14 & "Pisum sativum Linn. (Seeds, Split) | Patnai" & Pisum sativum L., Fabaceae (seeds) \\
\hline 15 & "Persian Almond 1869” & Prunus dulcis (Mill.) D.A.Webb., Rosaceae (fruits) \\
\hline 16 & Garnet & Punica granatum L., Lythraceae (fruits) \\
\hline 17 & “Fructus Rubi idaei” & Rubus idaeus L., Rosaceae (fruits) \\
\hline 18 & "Sea Kale” & $\begin{array}{l}\text { Saccharina latissima (L.) C. E. Lane, C. Mayers, Druehl, et G. } \\
\text { W. Saunders, Laminariaceae (thallus) }\end{array}$ \\
\hline 19 & "Rye spikes from Minsk province in 1875” & Secale cereale L., Poaceae (fruits) \\
\hline 20 & "Sesamum indicum Linn. (white)" & Sesamum indicum L., Pedaliaceae (seeds) \\
\hline 21 & "Potato Solanum tuberosumtuber sprouted190..." & Solanum tuberosum L., Solanaceae (root vegetables) \\
\hline 22 & “Spinacea oleracea Mill. (Leaves)” & Spinacia oleracea L., Asteraceae (leaves) \\
\hline 23 & “Semen Cacao Guajaguit” & Theobroma cacao L., Malvaceae (seeds) \\
\hline 24 & “Zea Mays Linn. (Grain)” & Zea mays L., Poaceae (fruits) \\
\hline \multicolumn{3}{|c|}{ Spices, species, fragrances } \\
\hline 25 & "Bulbus Allii (garlic)" & Allium sativum L., Amaryllidaceae \\
\hline 26 & "Peucedanum graveolens Benth. (Seeds)" & Anethum graveolens L.(fruits), Apiaceae \\
\hline 27 & “Apium graveolens Linn. (Seeds.)” & Apium graveolens L., Apiaceae (fruits) \\
\hline
\end{tabular}



Petersburg Chemical Pharmaceutical University, Which Can Be Used in Food

(Table 1 continued)

\begin{tabular}{|c|c|c|}
\hline № & Sample name & $\begin{array}{l}\text { Names of species and family } \\
\text { (part or product is indicated in brackets) }\end{array}$ \\
\hline 28 & "Herba Abrotani” & Artemisia abrotanum L., Asteraceae (grass) \\
\hline 29 & “Herba Dracunculi” & Artemisia dracunculus L., Asteraceae (grass) \\
\hline 30 & "Semen Sinapis Sarepta” & $\begin{array}{l}\text { Brassica juncea (L.)Vassiliĭ Matveievitch Czernajev, } \\
\text { Brassicaceae (seeds) }\end{array}$ \\
\hline 31 & “Moutarde in grains” & Brassica nigra L., Brassicaceae (seeds) \\
\hline 32 & “Capparis spinosa | Fruits | [Tokhmi kyava]” & Capparis spinosa L., Capparidaceae (fruits) \\
\hline 33 & “Piper Cayennae Chiliae” & Capsicum annuum L., Solanaceae (fruits) \\
\hline 34 & “Fructus Carvi Russisch” & Carum carvi L., Apiaceae (fruits) \\
\hline 35 & “Cortex Cin̄amom” & Cinnamomum verum J. Presl, Lauraceae (bark) \\
\hline 36 & "Lime. (Shell)" & Citrus $\times$ aurantifolia (Christm.) Swingle(peel), Rutaceae \\
\hline 37 & "Fructus Auranthii immaturi Ph. G. IV.” & Citrus aurantium L.(unripefruits), Rutaceae \\
\hline 38 & "Citrus aurantium var. sinensis | Orange" & Citrus $\times$ aurantium L.(fruits), Rutaceae \\
\hline 39 & Lemon fruits & Citrus limon (L.) Osbeck(fruits), Rutaceae \\
\hline 40 & “Bergamot” & Citrus bergamia Risso, Rutaceae (fruits) \\
\hline 41 & "Fructus Coriandri Russisch” & Coriandrum sativum L., Apiaceae (fruits) \\
\hline 42 & "Fructus Cumini depurati” & Cuminum cyminum L., Apiaceae (fruits) \\
\hline 43 & "Curcuma aromatica Salisb. (Rhizoma)” & Curcuma aromatica Salisb., Zingiberaceae (rhizomes) \\
\hline 44 & "Curcuma longa Roxb. (Turmeric.)” & Curcuma longa L., Zingiberaceae (rhizomes) \\
\hline 45 & “Amomum cardamomum L.”“Bitter seeded Cardamon” & Elettaria cardamomum (L.) Maton, Zingiberaceae (fruits) \\
\hline 46 & “Fructus Foeniculi russisch” & Foeniculum vulgare Mill., Apiaceae (fruits) \\
\hline 47 & “Fructus Anisi stellati” & Illicium anisatum L., Schizandraceae (fruits) \\
\hline 48 & "Folia Lauri nobilis" & Laurus nobilis L., Lauraceae (leaves) \\
\hline 49 & “Herba Melissae” & Melissa officinalis L., Lamiaceae (grass) \\
\hline 50 & “Herba Menthae piperit.” & Mentha $\times$ piperita L., Lamiaceae (grass) \\
\hline 51 & "Sem. Myristicae aus Nuces moschatae" & Myristica fragrans Houtt., Myristicaceae (seeds) \\
\hline 52 & “Herba Basilici” & Ocimum basilicum L., Lamiaceae (grass) \\
\hline 53 & "Herba Majoranae” & Origanum majorana L., Lamiaceae (grass) \\
\hline 54 & "Eugenia Pimenta | Englihse Gewurz | English Pepper” & Pimenta dioica (L.) Merr., Myrtaceae (fruits) \\
\hline 55 & "Fructus Anisi Bulgarisch” & Pimpinella anisum L., Apiaceae (fruits) \\
\hline 56 & “Piper nigrum Saygon” & Piper nigrum L., Piperaceae (fruits) \\
\hline 57 & "Folia Rosmarini” & Rosmarinus officinalis L., Lamiaceae (leaves) \\
\hline 58 & "Herba Saturejae" & Satureja hortensis L., Lamiaceae (grass) \\
\hline 59 & "Baies roses de l'Ile Maurice" & Schinus terebinthifolius Raddi, Anacardiaceae (fruits) \\
\hline 60 & “Caryophyllus aromaticus | Krüntuelken” & Syzygium aromaticum (L.) Merr. \& Perry, Myrtaceae (buds) \\
\hline 61 & "Thymi Ph. G. IV. gallica in foliis” & Thymus vulgaris L., Lamiaceae (grass) \\
\hline 62 & “Trigonella Fœnum-græcum Linn. (Seeds)” & Trigonella foenum-graecum L., Fabaceae (seeds) \\
\hline 63 & “Fructus Vanilla” & Vanilla planifolia Jacks. ex Andrews, Orchidaceae (fruits) \\
\hline \multicolumn{3}{|c|}{ Raw materials for preparing drinks } \\
\hline 64 & “Thea bohea 1843 и 1893” & Camellia sinensis (L.) Kuntze, Theaceae (leaves) \\
\hline 65 & "Rad. Cychorii | Estl. prov. 1915” & Cichorium intybus L., Asteraceae (root vegetables) \\
\hline 66 & "Coffea arabica | fruits” & Coffea arabica L., Rubiaceae (seeds) \\
\hline 67 & “Coffea ex Aruricu 1874” & Coffea sp., Rubiaceae (seeds) \\
\hline 68 & "Semen Colae siccatae" & $\begin{array}{l}\text { Cola acuminata Schott \& Endl. или C. nitida L., Malvaceae } \\
\text { (seeds) }\end{array}$ \\
\hline 69 & “Fructus Lupuli” & Humulus lupulus L., Cannabaceae (fruits) \\
\hline \multicolumn{3}{|c|}{ Raw materials for obtaining oil } \\
\hline 70 & "Linum usitatissimum Linn. (Seeds, red)" & Linum usitatissimum L., Linaceae (seeds) \\
\hline 71 & “Semen Pruni Persicae” & Prunus persica (L.) Batsch, Rosaceae (fruits) \\
\hline
\end{tabular}


(Table 1 continued)

\begin{tabular}{|c|c|c|}
\hline № & Sample name & $\begin{array}{l}\text { Names of species and family } \\
\text { (part or product is indicated in brackets) }\end{array}$ \\
\hline \multicolumn{3}{|c|}{ Food colorings } \\
\hline 72 & "Bixa Orellana Linn. (Seeds.)” & Bixa orellana L., Bixaceae (seeds) \\
\hline 73 & “Cochenillae Mexico | Ph. 1856” & Dactylopius coccus Costa, Dactylopiidae (whole) \\
\hline 74 & "Indigo Tiflis” & Indigofera tinctoria L., Fabaceae (indigo) \\
\hline \multicolumn{3}{|c|}{ Emulgators and gel-formers } \\
\hline 75 & "Gelatinae alba et rubra" & Bovinae (gelatin) \\
\hline 76 & “Lichen Caragaheen” & Chondrus crispus Stackh., Gigartinaceae (thallus) \\
\hline 77 & “Agar-agar” & $\begin{array}{l}\text { Phyllophora sp.,Gracilaria sp.,Gelidium sp.,Ceramium sp., } \\
\text { Rhodophyta(agar-agar)| }\end{array}$ \\
\hline \multicolumn{3}{|c|}{ Sweeteners } \\
\hline 78 & $\begin{array}{l}\text { "Bukhara licorice obtained in } 1925 \text { from the factory } \\
\text { "Licorice"”" }\end{array}$ & Glycyrrhiza glabra L., Fabaceae (roots) \\
\hline 79 & “Glycyrrhiza uralensis” & Glycyrrhiza uralensis Fisch. ex DC., Fabaceae (roots) \\
\hline
\end{tabular}

Table 2 Non-commonly used raw materials.

\begin{tabular}{|c|c|c|c|}
\hline № & Sample name & $\begin{array}{l}\text { Names of the species and family (in brackets - part or } \\
\text { product) }\end{array}$ & Link \\
\hline \multicolumn{4}{|c|}{ Custom raw materials } \\
\hline 1 & $\begin{array}{l}\text { "Hibiscus esculentus L. } \odot \text { (fructus ovalis) } \mid \text { Okrapods } \mid \\
\text { 1877” }\end{array}$ & Abelmoschus esculentus (L.) Moench, Malvaceae (fruits) & 6 \\
\hline 2 & “Aegle marmelos” & Aegle marmelos (L.) Correâ, Rutaceae (fruits) & 6 \\
\hline 3 & "Herba Alchemill. Vulg." & Alchemilla vulgaris L., Rosaceae (grass) & 4 \\
\hline 4 & “Alhagi camel | Manna on a camel thorn” & Alhagi maurorum Medik., Fabaceae (fruits) & 11 \\
\hline 5 & “Rad. Bardanae” & Arctium lappa L., Asteraceae & 4 \\
\hline 6 & "Artocarpus integrifolia Linn. (Unripe fruit)” & Artocarpus integer (Thunb) Merr.., Moraceae (fruits) & 6 \\
\hline 7 & "Benincasa cerifera Sary (Seeds)" & Benincasa hispida (Thunb.) Cogn., Cucurbitaceae (seeds) & 6 \\
\hline 8 & "Berberis heterophylla | Berries | Uzbek.*.” & Berberis microphylla G. Forst., Berberidaceae (fruits) & 11 \\
\hline 9 & “Barberry fruits | Karakol gorge, 1949” & Berberis sp., Berberidaceae (fruits) & 11 \\
\hline 10 & “Rhizoma Bergeniae” & Bergenia crassifolia (L.) Fritsch, Saxifragaceae & 4 \\
\hline 11 & “Seidencorons” & Bombyx mori L., Bombycidae (butterfly larvae in cocoons) & 3 \\
\hline 12 & "Herba Borraginis" & Borago officinalis L., Boraginaceae (grass) & 4 \\
\hline 13 & “Cajanus indicus Spreng (Seeds, Variety)” & Cajanus cajan (L.) Millsp., Fabaceae (seeds) & 11 \\
\hline 14 & $\begin{array}{l}\text { “[So-ga-ba] | Capsella bursa pastoris L. | Grass | } \\
\text { Transbaikalian expedition VIEM 1933” }\end{array}$ & Capsella bursa-pastoris (L.) Medik., Brassicaceae (grass) & 4 \\
\hline 15 & “Hikoria Pecan from Sukhum1929” & $\begin{array}{l}\text { Carya illinoiensis (Wangenh.) K. Koch, Juglandaceae } \\
\text { (fruits) }\end{array}$ & 10 \\
\hline 16 & “Carya porcina N. York” & Caryaglabra (Mill.) Sweet., Juglandaceae (fruits) & 10 \\
\hline 17 & "Edible chestnut | Castanea vesca | 1871” & Castanea vesca Gaertn., Fagaceae (fruits) & 10 \\
\hline 18 & “Ceratonia | Monte Carlo” & Ceratonia siliqua L., Fabaceae (fruits) & 11 \\
\hline 19 & "Lichen islandicus Ph. G. IV. erectus" & Cetraria islandica (L.) Ach., Parmeliaceae (thallus) & 4 \\
\hline 20 & "Cicer arietinum Linn. (Seeds, split) | var: Patnai” & Cicer arietinum L., Fabaceae (seeds) & 6 \\
\hline 21 & "Semen Citrulli" & $\begin{array}{l}\text { Citrullus lanatus (Thunb.) Matsum. \& Nakai, } \\
\text { Cucurbitaceae (seeds) }\end{array}$ & 6 \\
\hline 22 & "Lichen rangiferi” & $\begin{array}{l}\text { Cladonia rangiferina (L.) Weber ex F.H.Wigg., } \\
\text { Cladoniaceae (thallus) }\end{array}$ & 4 \\
\hline 23 & "Herba Cochleariae” & Cochlearia officinalis L., Brassicaceae (grass) & 11 \\
\hline 24 & "Bird nests India” & Callocalia sp. (nests) & 3 \\
\hline 25 & "Voloshsky nuts (overseas)Corylus tubulosa?” & Corylus maxima Mill, Betulaceae (fruits) & 10 \\
\hline 26 & $\begin{array}{l}\text { "[Da-dreg] | Cotoneaster vulgaris L. var. melanocarpa | } \\
\text { "Fruits | Atsagat. Transbaikalian expedition VIEM } \\
\text { 1933” }\end{array}$ & Cotoneaster integerrimus Medik., Rosaceae (fruits) & 4 \\
\hline
\end{tabular}



Petersburg Chemical Pharmaceutical University, Which Can Be Used in Food

(Table 2 continued)

\begin{tabular}{|c|c|c|c|}
\hline № & Sample name & $\begin{array}{l}\text { Names of the species and family (in brackets - part or } \\
\text { product) }\end{array}$ & Link \\
\hline 27 & "Crataegus coccinea" & Crataegus coccinea L., Rosaceae (fruits) & 11 \\
\hline 28 & “Semen Meloni” & Cucumis melo L., Cucurbitaceae (seeds) & 6 \\
\hline 29 & "Semen Cucurbitae" & Cucurbita pepo L., Cucurbitaceae (seeds) & 6 \\
\hline 30 & "Cyperus rotundus Linn. (Roots)” & Cyperus rotundus L., Cyperceae (root vegetables) & 12 \\
\hline 31 & “Diospyros lotus | Sptbr 69.” & Diospyros lotus L., Ebenaceae (fruits) & 11 \\
\hline 32 & "Diospyros Embryopteris Pers. (Fruit)" & Diospyros malabarica (Desr.) Kostel., Ebenaceae (fruits) & 11 \\
\hline 33 & “Herba Spiraeae ulmariae” & Filipendula ulmaria (L.) Maxim., Rosaceae (grass) & 4 \\
\hline 34 & “Spiraea filipendula 1869” & Filipendula vulgaris Moench, Rosaceae & 4 \\
\hline 35 & $\begin{array}{l}\text { "Manna cannulata | Fraxinus ornus | Oleaceae | } \\
\text { Mittelmeergebiet" }\end{array}$ & Fraxinus ornus L., Oleaceae (manna) & 11 \\
\hline 36 & “Helvella esculenta | Brain mushroom 1908” & $\begin{array}{l}\text { Gyromitra esculenta (Pers. Ex Pers.) Fr., Discinaceae } \\
\text { (fruiting bodies) }\end{array}$ & 7 \\
\hline 37 & "Nuces Juglandis regiae immat" & Juglans regia L., Juglandaceae (fruits) & 10 \\
\hline 38 & "Fructus Juniperi Ph. G. IV. germanici | italici” & Juniperus communis L., Cupressaceae (fruits) & 4 \\
\hline 39 & “Dolichos lablab” & Lablab purpureus (L.) Sweet, Fabaceae (seeds) & 6 \\
\hline 40 & “Lactarius rufus, Scop.” & Lactarius rufus (Scop.) Fr.,Russulaceae (fruiting bodies) & 7 \\
\hline 41 & Lactarius torninosus & $\begin{array}{l}\text { Lactarius torminosus (Schaeff.) Pers.,Russulaceae (fruiting } \\
\text { bodies) }\end{array}$ & 7 \\
\hline 42 & "Lagenaria vulgaris Seringe. (Seeds)" & $\begin{array}{l}\text { Lagenaria siceraria (Molina) Standl., Cucurbitaceae } \\
\text { (seeds) }\end{array}$ & 6 \\
\hline 43 & “Lecanora esculenta” & Lecanora esculenta (Pall.) Eversm., Lecanoraceae (thallus) & 7 \\
\hline 44 & “Pyrus prunifolia | Octbr. 77.” & $\begin{array}{l}\text { Malus } \times \text { prunifolia (Willd.) Borkh.var. prunifolia, } \\
\text { Rosaceae (fruits) }\end{array}$ & 11 \\
\hline 45 & $\begin{array}{l}\text { "Jatropha Manihot | } \\
\text { "Sago “Tapiokka”” }\end{array}$ & Manihot esculenta Crantz, Euphorbiaceae (sago) & 11 \\
\hline 46 & “[Kumien]” & Mangifera indica L., Anacardiaceae(seeds) & 6 \\
\hline 47 & “Mespilus” & Mespilus germanica L., Rosaceae (fruits) & 11 \\
\hline 48 & "Mimusops Elengi Linn. (Fruit.)" & Mimusops elengi L., Sapotaceae (fruits) & 11 \\
\hline 49 & “Morus alba | Aug 1879” & Morus alba L., Moraceae (fruits) & 10 \\
\hline 50 & “Nymphaea Lotus Linn. (Seeds)” & Nelumbo nucifera Gaertn.,Nelumbonaceae (seeds) & 10 \\
\hline 51 & “Radix Salep Moscau” & Orchis sp.,Dactilorrhiza sp., Orchidaceae (root vegetables) & 4 \\
\hline 52 & "Peony dodging roots" & Paeonia anomala L., Paeoniaceae (roots) & 4 \\
\hline 53 & “Papaver somniferum Linn. (Seeds)” & Papaver somniferum L., Papaveraceae (seeds) & 10 \\
\hline 54 & “Paspalum scrobiculatum Linn. (Seeds)” & Paspalum scrobiculatum L., Poaceae (fruits) & 12 \\
\hline 55 & "Pennisetum typhoideum Rich. (Seeds.)" & Pennisetum glaucum (L.) R. Br., Poaceae (fruits) & 12 \\
\hline 56 & "Phyllanthus Emblica Linn. (Fruit.)” & Phyllanthus emblica L., Phyllanthaceae (fruits) & 11 \\
\hline 57 & “Fructus Alkekengi” & Physalis alkekengi L., Solanaceae (fruits) & 11 \\
\hline 58 & $\begin{array}{l}\text { "Cones of Siberian cedar | Pinus Cembra L. |on the } \\
\text { outskirts of Barnaul | sent d-r MD Dumberg”" }\end{array}$ & Pinus cembra L., Pinaceae (fruits) & 9 \\
\hline 59 & $\begin{array}{l}\text { "Pinus Gerardiana Wall. (Fruit.)”"'Pinus Gerardiana } \\
\text { Wall. (Fruit.)” }\end{array}$ & Pinus gerardiana Wall. Ex D.Don., Pinaceae (fruits) & 9 \\
\hline 60 & “Folium Primulae officinalis” & Primula veris L., Primulaceae (leaves) & 4 \\
\hline 61 & “Armeniaca sibirica | Stone plum” & Prunus sibirica L., Rosaceae (fruits) & 4 \\
\hline 62 & “Fructus Pruni Padi” & Prunus padus L., Rosaceae (fruits) & 4 \\
\hline 63 & “Herba Pulmonariae maculosae” & Pulmonaria officinalis L., Boraginaceae (grass) & 4 \\
\hline 64 & “Rhizopogon albus | Earthen truffle1877” & $\begin{array}{l}\text { Rhizopogon albus (Bull.) Fr., Rhizopogonaceae (fruiting } \\
\text { bodies) }\end{array}$ & 7 \\
\hline 65 & “dogrose spiny” & Rosa acicularis Lindl., Rosaceae (fruits) & 4 \\
\hline 66 & "Fructus Cynocbati sine semina electi” & Rosa canina L., Rosaceae (fruits) & 11 \\
\hline 67 & “dog rose Dahuri” & Rosa davurica Pall., Rosaceae (fruits) & 11 \\
\hline 68 & “Rosa laevigata Michx | DRV. Hanoi.|X. 1963” & Rosa laevigata Michx., Rosaceae (fruits) & 11 \\
\hline
\end{tabular}


Petersburg Chemical Pharmaceutical University, Which Can Be Used in Food

(Table 2 continued)

\begin{tabular}{|c|c|c|c|}
\hline № & Sample name & $\begin{array}{l}\text { Names of the species and family (in brackets - part or } \\
\text { product) }\end{array}$ & Link \\
\hline 69 & "rose hips wrinkled" & Rosa rugosa Thunb., Rosaceae (fruits) & 11 \\
\hline 70 & "Rubus ellipticus Smith (Fruits)” & Rubus ellipticus Sm., Rosaceae (fruits) & 11 \\
\hline 71 & $\begin{array}{l}\text { “[Sho-mang]| Rumex acetosa L. | Grass. Atsagat. } \\
\text { Transbaikalian expedition VIEM 1933” }\end{array}$ & Rumex acetosa L., Polygonaceae (grass) & 4 \\
\hline 72 & “Saccharum officinarum "Blüthen” & Saccharum officinarum L., Poaceae (grass) & 12 \\
\hline 73 & “Fructus Sambuci sicccati” & Sambucus nigra L., Adoxaceae (fruits) & 11 \\
\hline 74 & "Fructus Schisandrae" & $\begin{array}{l}\text { Schizandra chinensis (Turcz.) Baill., Schizandraceae } \\
\text { (fruits) }\end{array}$ & 10 \\
\hline 75 & “Serpula lacrymans” & $\begin{array}{l}\text { Serpula lacrymans (Wulfen) Schroet., Serpulaceae (fruiting } \\
\text { bodies) }\end{array}$ & 7 \\
\hline 76 & “Setaria italica Kunth. (Seeds)” & Setaria italica (L.) P. Beauv., Poaceae (fruits) & 12 \\
\hline 77 & $\begin{array}{l}\text { “[Syucy] from the Chinese city San-sin. Sorghum } \\
\text { 1856.” }\end{array}$ & Sorghum bicolor (L.) Moench., Poaceae (fruits) & 12 \\
\hline 78 & “Spondias mangifera Pers. (Fruit)” & Spondias pinnata (L. f.) Kurz, Anacardiaceae (fruits) & 11 \\
\hline 79 & "Boletus luridus Krubuk | 190... г." & $\begin{array}{l}\text { Suillus luridus (Schaeff.) Murrill, Boletaceae (fruiting } \\
\text { bodies) }\end{array}$ & 7 \\
\hline 80 & “Beans Tamarindus indica 1909” & Tamarindus indica L., Fabaceae (fruits) & 6 \\
\hline 81 & “Trapa natans | Water chestnut| 1915” & $\begin{array}{l}\text { Trapa natans var. bispinosa (Roxb.) Makino, Lythraceae } \\
\text { (root vegetables) }\end{array}$ & 4 \\
\hline 82 & “Trapa spinosa Roxb. (Nuts.)” & Trapa natans var. bispinosa (Roxb.) Makino, Lythraceae & 4 \\
\hline 83 & “Typha angustifolia” & Typha angustifolia L., Typhaceae & 4 \\
\hline 84 & "Herba Urticae” & Urtica dioica L., Urticaceae (grass) & 4 \\
\hline 85 & "Fructus Myrtilli siccati” & Vaccinium myrtillus L., Ericaceae (fruits) & 4 \\
\hline 86 & “Fructus Viburni opuli” & Viburnum opulus L., Adoxaceae (fruits) & 4 \\
\hline 87 & "Phaseolus aconitifolius Jacq (Seeds)" & Vignaaconitifolia (Jacq.) Marechal, Fabaceae (seeds) & 6 \\
\hline 88 & $\begin{array}{l}\text { "Phaseolus Mungo Linn. Var. Radiatus (Seeds, Split, } \\
\text { Varierty)” }\end{array}$ & Vigna mungo (L.) Hepper, Fabaceae (seeds) & 6 \\
\hline 89 & "Vigna Catiang Endl. (Seeds)" & Vigna unguiculata (L.) Walp., Fabaceae (seeds) & 6 \\
\hline 90 & “Fructus Jujubae” & Zizyphus jujuba Mill., Rhamnaceae (fruits) & 6 \\
\hline 91 & “Zizyphus mauritiana fruits | [Chipan]” & Zizyphus mauritiana Lam., Rhamnaceae (fruits) & 6 \\
\hline \multicolumn{4}{|c|}{ Spices, species, fragrances } \\
\hline 92 & "Rhizoma Calami Ph. G. IV. mundatur albissim" & Acorus calamus L., Acoraceae & 4 \\
\hline 93 & “Amomum Korarima Pereira” & Aframomum cororrima (A.Braun) P.C.M. Jansen (fruits) & 13 \\
\hline 94 & $\begin{array}{l}\text { "Fructus Cardamomi Ph. G. IV. Malabarici electi } \\
\text { excorticati” }\end{array}$ & Aframomum melegueta K. Schum., Zingiberaceae (fruits) & 12 \\
\hline 95 & “Radix Victorialis longa” & Allium victorialis L., Amaryllidaceae (bulbs) & 4 \\
\hline 96 & $\begin{array}{l}\text { "Chinese Ginger [Dzyanch] | Alpinia galangal |, } \\
\text { Shanghai 1891. } 30 \text { / VIII | from V. Tikhomirov" }\end{array}$ & Alpinia galanga (L.) Willd., Zingiberaceae (rhizomes) & 12 \\
\hline 97 & $\begin{array}{l}\text { "Rhizoma Galangae | } \\
\text { Alpinia officinarum Hance” }\end{array}$ & Alpinia officinarum Hance, Zingiberaceae (rhizomes) & 12 \\
\hline 98 & “Amomum costatum Roxb. | DRV Hanoi” & Amomum costatum Roxb., Zingiberaceae (fruits) & 12 \\
\hline 99 & “Amomum xanthioides Wallich” & $\begin{array}{l}\text { Amomum villosum var. xanthioides (Wall. ex Baker) N.L. } \\
\text { Wu \& S.J. Chen, Zingiberaceae (fruits) }\end{array}$ & 12 \\
\hline 100 & “Amomum maximum Roxb.” & Amomum maximum Roxb., Zingiberaceae (fruits) & 12 \\
\hline 101 & "Radix Angelicae archangel." & Angelica archangelica L., Apiaceae & 4 \\
\hline 102 & "Rhizoma Curcumae rotunda” & $\begin{array}{l}\text { Boesenbergia rotunda (L.) Mansf., Zingiberaceae } \\
\text { (rhizomes) }\end{array}$ & 12 \\
\hline 103 & “Cortex Canellae albae” & Canella winterana (L.) Gaertn., Canellaceae & 10 \\
\hline 104 & “Cinnamomum obtusifolium (Leaves)" & $\begin{array}{l}\text { Cinnamomum bejolghota (Buch.-Ham.) Sweet(leaves), } \\
\text { Lauraceae }\end{array}$ & 10 \\
\hline 105 & “Cort. Cinnamomi casiae” & $\begin{array}{l}\text { Cinnamomum burmannii (Nees \& T.Nees) Blume, } \\
\text { Lauraceae (bark) }\end{array}$ & 5 \\
\hline 106 & “Cortex Culilawan” & Cinnamomum culilawan Blume, Lauraceae (bark) & 10 \\
\hline
\end{tabular}



Petersburg Chemical Pharmaceutical University, Which Can Be Used in Food

(Table 2 continued)

\begin{tabular}{|c|c|c|c|}
\hline № & Sample name & $\begin{array}{l}\text { Names of the species and family (in brackets - part or } \\
\text { product) }\end{array}$ & Link \\
\hline 107 & “Cinnamomum malabathorum G. Don.” & $\begin{array}{l}\text { Cinnamomum malabatrum (Burm.f.) J. Presl, Lauraceae } \\
\text { (bark) }\end{array}$ & 10 \\
\hline 108 & “Cleome viscosa Linn. (Seeds)” & Cleome viscosa L., Cleomaceae (seeds) & 11 \\
\hline 109 & "Curcuma angustifolia Roxb. (Wild Arrow-Root)" & Curcuma angustifolia Roxb., Zingiberaceae (rhizomes) & 12 \\
\hline 110 & "Rhizoma Zedoariae luteae" & $\begin{array}{l}\text { Curcuma zedoaria (Christm.) Roscoe, Zingiberaceae } \\
\text { (rhizomes) }\end{array}$ & 12 \\
\hline 111 & "Gumir: Assae foetid: in granis” & Ferula assa-foetida L., Apiaceae (gum) & 5 \\
\hline 112 & "Macis ex Myristica tomentosa” & Horsfieldia tomentosa Warb., Myristicaceae (seeds) & 11 \\
\hline 113 & "Herba Hyssopii in foliis cum floribus" & Hyssopus officinalis L., Lamiaceae (grass) & 11 \\
\hline 114 & “Semen Nasturtii horten.” & Lepidium sativum L., Brassicaceae (seeds) & 13 \\
\hline 115 & "Radix Levistici” & Levisticum officinale Koch, Apiaceae (roots) & 11 \\
\hline 116 & "Kaempferia rotunda (Root)" & Kaempferia rotunda L., Zingiberaceae (rhizomes) & 12 \\
\hline 117 & “Kermes” & Kermes vermilio Planch., Kermesidae (whole) & 2 \\
\hline 118 & "Mentha arvensis Linn. (Plant)" & Mentha arvensis L., Lamiaceae (grass) & 5 \\
\hline 119 & “Herba Menthae citriod.” & Mentha citrata Ehrh., Lamiaceae (grass) & 13 \\
\hline 120 & "Herba Menthae Puleg.” & Mentha pulegium L., Lamiaceae (grass) & 5 \\
\hline 121 & "Herba Menthae crispae" & Mentha spicata L., Lamiaceae (grass) & 13 \\
\hline 122 & “Agaricus Scorodonius | 1874” & $\begin{array}{l}\text { Mycetinis scorodonius (Fr.) A.W. Wilson \& Desjardin, } \\
\text { Marasmiaceae (fruiting bodies) }\end{array}$ & 7 \\
\hline 123 & "Balsamum Tolutanum” & $\begin{array}{l}\text { Myroxylon balsamum var.balsamum (L.) Harms, Fabaceae } \\
\text { (balsam) }\end{array}$ & 5 \\
\hline 124 & “[Siya dana] | Nigella sativa | Seeds | Uzbek*.” & Nigella sativa L., Ranunculaceae (seeds) & 10 \\
\hline 125 & "Nymphaea alba. 24.VII.64.” & Nymphaea alba L., Nymphaeaceae(rhizomes) & 4 \\
\hline 126 & "Herba Origani cretici” & Origanum vulgare L., Lamiaceae (grass) & 11 \\
\hline 127 & "Radix Petroselini” & Petroselinum crispum (Mill.) Fuss, Apiaceae (roots) & 11 \\
\hline 128 & "Piper cubeba” & Piper cubeba L. f., Piperaceae (fruits) & 10 \\
\hline 129 & "Piper longum Linn. (Fruit, variety)" & Piper longum L., Piperaceae (fruits) & 6 \\
\hline 130 & “Piper Chaba Bl. (twigs)” & Piper retrofractum Vahl., Piperaceae (fruits) & 6 \\
\hline 131 & "Radix Sanguisorbae officinalis" & Sanguisorba officinalis(roots and rhizomes) & 4 \\
\hline 132 & "Baies roses de l'Ile Maurice" & Schinus terebinthifolius Raddi, Anacardiaceae (seeds) & 13 \\
\hline 133 & “Semen Erucae” & Sinapis alba L., Brassicaceae (seeds) & 13 \\
\hline 134 & "Fructus Sorbi aucupariae" & Sorbus aucuparia L., Rosaceae (fruits) & 11 \\
\hline 135 & “Semen Syzygii jambolani” & Syzygium cumini (L.) Skeels, Myrtaceae (buds) & 6 \\
\hline 136 & $\begin{array}{l}\text { “[Brag-boy]| Thymus serpyllum L. | Grass | Atsagat. } \\
\text { Transbaikalian expedition VIEM 1933” }\end{array}$ & Thymus serpyllum L., Lamiaceae (grass) & 11 \\
\hline 137 & "Carum copticum Benth. (Seeds)" & Trachyspermum ammi Sprag., Apiaceae (fruits) & 11 \\
\hline 138 & "Carum Roxburghianum Benth. (Seeds)" & $\begin{array}{l}\text { Trachyspermum roxburghianum (DC.) H. Wolff, Apiaceae } \\
\text { (fruits) }\end{array}$ & 13 \\
\hline 139 & "Vitex negundo Linn. (Fruits)" & Vitex negundo L., Vitex trifolia L., Lamiaceae (fruits) & 11 \\
\hline 140 & "Xanthoxylum Rhetsa” & Zanthoxylum rhetsa (Roxb.) DC, Rutaceae (fruits) & 11 \\
\hline \multicolumn{4}{|c|}{ Raw materials for preparing drinks } \\
\hline 141 & $\begin{array}{l}\text { "[Dug-mo-n'ung man-ba] | Chamaenerion (Epilobium) } \\
\text { angustifolium (L.) Scop. | Leaves | Transbaikalian } \\
\text { expedition VIEM 1933” }\end{array}$ & $\begin{array}{l}\text { Chamaenerion angustifolium (L.) Scop., Onagraceae } \\
\text { (leaves) }\end{array}$ & 4 \\
\hline 142 & "Folia Mate, Paraguay Tee” & Ilex paraguariensis A. St.-Hil., Aquifoliaceae (leaves) & 11 \\
\hline 143 & "Semina Paulliniae sorbilis (guarana) 1866" & Paullinia cupana Kunth., Sapindaceae (seeds) & 11 \\
\hline 144 & “Radix Taraxaci” & Taraxacum officinale Webb., Asteraceae & 4 \\
\hline \multicolumn{4}{|c|}{ Raw materials for obtaining oil } \\
\hline 145 & "Fructus Cannabis" & Cannabis sativa L., Cannbaceae (fruits) & 4 \\
\hline 146 & "Fructus Platani orientale” (label wrong!) & Fagus orientalis Lipsky, Fagaceae (fruits) & 10 \\
\hline
\end{tabular}


Petersburg Chemical Pharmaceutical University, Which Can Be Used in Food

(Table 2 continued)

\begin{tabular}{|c|c|c|c|}
\hline № & Sample name & $\begin{array}{l}\text { Names of the species and family (in brackets-part or } \\
\text { product) }\end{array}$ & Link \\
\hline 147 & "Fructus Madiae sativae” & Madia sativa Molina, Asteraceae (fruits) & 11 \\
\hline \multicolumn{4}{|c|}{ Sources of starch } \\
\hline 148 & "Radix Aronis" & Arum maculatum L., Araceae (tubers) & 12 \\
\hline 149 & “Radix Cannae” & Canna sp., Cannaceae (tubers) & 12 \\
\hline 150 & “Amylum Maranth” & Marantha arundinacea L., Maranthaceae (starch) & 12 \\
\hline 151 & “Sago aus China” & Metrxylon sagu Rottb., Arecaceae (sago) & 12 \\
\hline \multicolumn{4}{|c|}{ Emulgators and gel-formers } \\
\hline 152 & $\begin{array}{l}\text { "Gummitragant technical } 1 \text { grade. Sorting } 1935 \text { Baku } \\
\text { base V/O "LTS”” }\end{array}$ & Astragalus tragacantha L., Fabaceae (gum) & 11 \\
\hline 153 & "Gummi arabicum in granis” & Vachellia sp., Fabaceae (gum) & 5 \\
\hline \multicolumn{4}{|c|}{ Sweeteners } \\
\hline 154 & "Radix Polypodii" & Polypodium vulgare L., Polypodiaceae (rhizomes) & 8 \\
\hline 155 & “Herba Lippiae mexicanae” & Phyla dulcis (Trevir.) Moldenke, (grass) & 13 \\
\hline
\end{tabular}

Note. Uzbek.*-short for Uzbekistan.

Totally, 254 units considered of the collection of medicinal raw materials of the Department of Pharmacognosy of the St. Petersburg Chemical-Pharmaceutical University, which is about $20 \%$ of the total number of non-repeated samples. Thus, a substantial part of this collection (which has a predominantly medical orientation) can also be used as food materials. In all cases (and for conventionally commonly used raw materials, and for conventionally not commonly used) about a half of the samples can be used both as food and as a medicine are a group of spices, species and aromatic plants.

\section{References}

Klemper, A. V., Belodubrovskaya, G. A., and Luzhanin, V. G. 2017. "On the Collection of Medicinal Plant Raw Materials of the Pharmacognosy Department of SPCP.”
III Gammerman Readings. SPb.: Publishing House SPKhFA: 25-27.

[1] Animal life (Volume 3). 1969. Moscow: Prosvestchenie.

[2] Animal life (Volume 5). 1970. Moscow: Prosvestchenie.

[3] Cherepnin, V. L. 1987. Food Plants of Siberia. Novosibirsk: "Nauka" (Siberian branch).

[4] Murav'eva, D. A. 1983. Tropical and Subtropical Medicinal Plants. Moscow: Medicine.

[5] Bernd, N., and Schulz, B. 2002. Tropical Fruits. Moscow: BMM AO.

[6] Plant life (Volume 2). 1976. Moscow: Prosvestchenie.

[7] Plant life (Volume 3). 1977. Moscow: Prosvestchenie.

[8] Plant life (Volume 4). 1978. Moscow: Prosvestchenie.

[9] Plant life (Volume 5, Part 1). 1980. Moscow: Prosvestchenie.

[10] Plant life (Volume 5, Part 2). 1981. Moscow: Prosvestchenie.

[11] Plant life (Volume 6). 1982. Moscow: Prosvestchenie.

[12] Ravindran, P. N. 2016. The Encyclopedia of Herbs and Species (Volumes 1, 2). Boston: CAB International. 